\title{
IMPUESTO A LA RENTA EN LA ECONOMÍA DIGITAL. ¿CUÁL ES LA SITUACIÓN DEL ECUADOR?
}

Income tax in the digital economy. What is the situation in Ecuador?

\author{
Jaime Pazmiño Jara \\ Estudio Jurídico Prado, Quito, Ecuador
}

\section{Resumen}

La transformación digital de los negocios es cada día más recurrente y tiene mayor incidencia en la economía mundial, por tanto, los desafíos que ésta representa para el ámbito tributario son innegables. Este artículo analiza los retos y riesgos en el impuesto a la renta que representan los modelos de negocio digitales, cuáles son las soluciones que se están desarrollando a nivel internacional, cuál es la situación del Ecuador frente a la tributación, la economía digital y qué estrategias podría implementar.

\section{Palabras clave}

Economía digital, Tributación internacional, Impuestos directos, Impuesto a la renta, Establecimiento permanente.

\begin{abstract}
Digital transformation of business is increasingly recurrent and has a greater impact on the world economy and therefore the challenges it represents for taxation purposes are undeniable. This article analyzes the challenges and risks that digital business models represent, what are the solutions that are being developed at an international level, what is the situation of Ecuador regarding taxation of the digital economy and what strategies could be implemented.
\end{abstract}

\section{Keywords}

Digital economy, International taxation, Direct taxes, Income tax, Permanent establishment.

\section{Introducción}

La transformación digital de los modelos de negocio en la economía actual ha cambiado la forma en la que los consumidores acceden a bienes y servicios, así como las condiciones del mercado actual para todos los agentes económicos.

La inclusión de la tecnología en el modelo de negocio de los agentes económicos ha provocado un aumento de las transacciones comerciales a nivel mundial, pues la incorporación de ésta por parte de los agentes económicos ha facilitado la producción y comercialización

\footnotetext{
${ }^{1}$ Abogado del área tributaria del Estudio Jurídico Prado. Candidato a Máster en Dirección y Administración de Empresas por la Universidad de La Rioja y Candidato a Magister en Derecho Tributario por la Universidad Andina Simón Bolívar(Ecuador). EspecialistaSuperior en DerechodelaEmpresaporlaUniversidad AndinaSimón Bolívar(Ecuador). Abogado por la Universidad San Francisco de Quito. Correo electrónico: jpazmino@ejprado.com; ORCID: https://orcid.org/0000-0002-1181-5243
} 
de bienes o prestación de servicios a nivel internacional. Incluso a raíz de la pandemia por efectos del Covid-19, a nivel mundial se ha incrementado la oferta de bienes y servicios a través de modelos de negocios de economía digital, así como la demanda, pues los usuarios han incrementado exponencialmente el uso de los mismos. El desarrollo de la economía digital en el Ecuador ha sido paulatino en comparación a otros países de la región y del mundo, por lo que ni la normativa vigente ni la jurisprudencia en la materia han analizado todos los efectos que jurídicamente pueden tener este tipo de modelos de negocios dentro de distintas áreas, en lo principal, los que se generan para la imposición de la renta.

El presente artículo analiza los retos y riesgos que la economía digital presenta para la tributación y cuáles son los mecanismos que el Ecuador dispone para enfrentar los retos de la economía digital y mitigar los riesgos a la luz de la normativa aplicable en nuestro ordenamiento jurídico, respecto a la aplicación de impuesto a la renta. Para ello, en primer lugar, se desarrollará el concepto de economía digital, los retos y riesgos tributarios de la misma con relación al impuesto a la renta y cuál es su impacto en el Ecuador.

\section{2. ¿Qué es la economía digital y cuál es la situación del Ecuador?}

Se entiende a la economía digital como aquella que se fundamenta en el internet y las tecnologías de la información para la implementación de un modelo de negocio. En términos de la Organización para la Cooperación y el Desarrollo Económico (en adelante, OCDE), la economía digital está caracterizada, en lo principal, por su dependencia en intangibles, datos personales, modelos de negocio multilaterales y por la dificultad de determinar dónde se crea valor (OCDE, 2015, p.16).

La injerencia de la tecnología ha afectado a varios sectores de la economía tradicional, en la forma en cómo ejercen su actividad económica, como educativo, servicios financieros, salud, entre otros; así, incluye todas aquellas transacciones comerciales que utilizan la tecnología para la venta de un bien o la prestación de un servicio, como, por ejemplo, todas las relativas al comercio electrónico.

La transformación digital de los negocios ha generado un cambio de paradigma tradicional respecto al comercio. Así, los modelos de negocio de economía digital dejan de depender de la presencia física tanto de su personal como de sus activos para la ejecución del mismo, así como para la relación con sus clientes. A lo anterior, debe considerarse que este tipo de modelos de negocio tienen la característica de multilateralidad, por medio de la cual el negocio conecta a más de un agente del mercado, interconectando los efectos entre ambos y utilizando toda la información que recaban de éstos a efectos de obtener mayores ventajas competitivas. (Massachusetts Institute of Technology, 2014).

En Ecuador, el desarrollo de la economía digital ha tardado más que en otros países o regiones, y de igual forma no ha sido objeto de mayor regulación por parte del ordenamiento jurídico nacional. En el año 2002, la Ley de Comercio Electrónico, Firmas Electrónicas y Mensajes de Datos introdujo la primera definición de comercio electrónico dentro de la disposición general novena, estableciéndolo como "toda transacción comercial realizada en parte o en su totalidad, a través de redes electrónicas de información”.

A pesar de esto, en el año 2019, con la expedición del nuevo Código de Comercio, se incluyó una definición normativa más clara y amplia sobre el comercio electrónico, así como otras normas relacionadas a la contratación dentro de una economía digital. El artículo 74 del Código de Comercio establece lo siguiente: "Art. 74.- Comercio electrónico es toda transacción comercial de bienes o servicios digitales o no, realizada en parte o en su totalidad a través de sistemas de información o medios electrónicos, considerando los tipos de relaciones existentes." 
Sin perjuicio de la norma citada, la regulación sobre comercio electrónico y economía digital es casi inexistente, considerando que, en el Ecuador, según la Cámara Ecuatoriana de Comercio Electrónico (CECE), las transacciones de comercio electrónico del año 2019 fueron de 24 millones, creciendo en un $42 \%$ en comparación con el año 2018. Este crecimiento significa que, en nuestro país, las transacciones de este tipo ascienden a 1.648 millones de dólares (Universidad Espíritu Santo, 2019).

A pesar de que la economía digital, y en específico el comercio electrónico, se encuentren en desarrollo y en un creciente aumento en nuestro país, el estudio de la CECE evidencia que los beneficiarios de los ingresos generados por las adquisiciones a través de canales electrónicos corresponden a portales internacionales (Universidad Espíritu Santo, 2019), lo cual implica varios retos y riesgos de índole tributario que se analizará en las siguientes secciones.

Con base en estas consideraciones iniciales, es necesario analizar cuáles son los retos y riesgos que la economía digital trae para los Estados y sus administraciones tributarias, y de manera específica cuáles cobran mayor incidencia dentro del análisis del impuesto a la renta.

\section{Principales problemas de la tributación en la economía digital}

En el año 2013, la OCDE adoptó la iniciativa para evitar la erosión de bases imponibles y el traslado de beneficios (en adelante, BEPS por sus siglas en inglés), ${ }^{2}$ la acción uno de dicho plan corresponde al análisis y medidas para contrarrestar los retos tributarios que se presentan por efectos de la transformación digital de los negocios. En este sentido, se han identificado varios retos relacionados a la aplicabilidad de los principios tradicionales de tributación internacional, la erosión de bases imponibles y el traslado de beneficios por efectos de un negocio digital, así como respecto a la aplicación de impuestos directos e indirectos dentro de una economía de estas características.

En lo principal, la economía digital trae retos respecto de la erosión de bases imponibles y el traslado de beneficios, entendiendo por esto, a los efectos impositivos que tienen las estructuras societarias complejas que buscan utilizar lagunas normativas o sistemas tributarios de ciertas jurisdicciones para tener una carga impositiva menor o inexistente. Así, las empresas con operaciones digitales a nivel transnacional de forma regular se domicilian en este tipo de jurisdicciones, sin presencia física alguna o nexo que las vincule a las jurisdicciones donde realizan sus operaciones u obtienen sus ingresos por medio de canales digitales.

Este tipo de estrategias, a la luz de los principios de la tributación internacional, permiten que estos modelos de negocio no tengan una tributación efectiva sobre las transacciones comerciales que realizan, situación que deriva en retos y riesgos para las Administraciones Tributarias en la imposición y recaudación tanto de impuestos directos como indirectos. ${ }^{3}$

Dentro del análisis de impuestos directos, el reporte final de la OCDE estableció que el principal riesgo de BEPS en este tipo de impuestos es la dificultad de tributación en el país en el que ocurre la transacción por la falta de presencia física de la empresa, inexistencia de imposición por medio de retenciones en la fuente, imposición nula o muy baja en el domicilio de la empresa y en el de los beneficiarios finales (OCDE, 2015).

\footnotetext{
${ }^{2}$ BEPS (Base erosion and profit shifting) por sus siglas en inglés, hace referencia a un plan de 15 acciones puntuales con la finalidad de tratar los problemas de erosión de la base imponible y al traslado de beneficios causados por multinacionales, con la finalidad de generar una menor o nula tributación de los beneficios por el uso de ciertas figuras legales o el aprovechamiento de lagunas de los sistemas tributarios de ciertas jurisdicciones.

${ }^{3}$ Los impuestos directos son aquellos que tienen como finalidad gravar el ingreso, el patrimonio o el capital con afectación directa a un sujeto pasivo, mientras que los impuestos indirectos son aquellos que gravan a un hecho como el consumo, trasladándose siempre al consumidor final.
} 
Adicionalmente, otro reto fundamental de la tributación de la economía digital es la forma en cómo los estados pueden exigir el cobro de los tributos sobre los sujetos de este tipo de modelos de negocio, pues al no tener presencia física la exigibilidad de los tributos se vuelve difícil de implementar.

Por otro lado, también se han identificado riesgos en la aplicación de impuestos indirectos, así por ejemplo, en la aplicación del impuesto al valor agregado en las transacciones comerciales por adquisición de bienes, servicios o intangibles; situación que cobran menor dificultad por la naturaleza de dicho impuesto y que han sido abordados por la legislación ecuatoriana. Sin perjuicio de esto, el presente análisis se centrará específicamente en aquellos riesgos en el impuesto a la renta o su equivalente en otras jurisdicciones, como se especifica a continuación.

\subsection{Riesgos en la tributación del impuesto a la renta}

De forma general, vale recordar que el impuesto a la renta es un impuesto directo mediante el cual se gravan los ingresos de fuente en un país, en un periodo fiscal determinado, puede ser sobre personas naturales (físicas) o sobre personas jurídicas. A la luz del derecho tributario internacional, así como de la normativa interna, corresponde analizar cuáles son los mecanismos que podría implementar una jurisdicción determinada para enfrentar los efectos de la economía digital sobre este tipo de impuestos directos.

Es importante partir de la potestad tributaria que tienen los Estados, que se entiende como aquella competencia de crear, modificar o extinguir tributos, así como de su aplicación y exigibilidad respecto a un sujeto dentro del territorio sobre el cual ejercen jurisdicción (Troya, 2014, pp. 120-121).

Las fuentes para la aplicación de la potestad tributaria de un Estado, tradicionalmente se divide en dos, las fuentes externas relacionadas a aquellas normas y principios de derecho tributario internacional, las cuales provienen principalmente de tratados internacionales y derecho comunitario, y tienen como finalidad regular aquellas relaciones que involucran distintas jurisdicciones; y, el ámbito interno respecto del cual la fuente principal es normativa local de cada país en el que se ejerce la potestad sobre todos aquellos sujetos residentes en dicho territorio (Borderas y Moles, 2008).

Ahora bien, la doctrina de tributación internacional establece dos reglas o principios respecto de qué jurisdicción es la que tiene el derecho para someter a imposición y por ende determinar qué Estado puede someter a su potestad tributaria la renta, siendo estos:

i. Principio personalista: determina que la jurisdicción que tendrá los derechos de tributación sobre una renta determinada, es aquella en la que la persona natural o jurídica sea residente. (Borderas y Moles, 2008, p. 27)

Un residente fiscal de un Estado puede ser determinado en función de su permanencia física o su nacionalidad, así como otros criterios que se pueden adoptar en función de la legislación interna de cada país.

Es decir, el principio personalista o también llamado de renta mundial, es aquel que somete a imposición la renta en función del sujeto y no de la fuente donde se generó la misma. Así, por ejemplo, Estados Unidos mantiene como principio para la imposición, el personalista, en el que se somete a tributación la renta obtenida por cualquier sujeto de dicha jurisdicción independientemente de la fuente en la que se obtenga. 
ii. Principio de fuente de la renta: determina que la jurisdicción que tendrá el derecho a someter a imposición la renta generada, será aquella en donde se generó la misma, independientemente de la residencia fiscal del sujeto pasivo. Así, los Estados que aplican este criterio someten a tributación toda aquella renta generada en su territorio. (Soto, 2017, p. 109)

Los principios referidos no son excluyentes. Existen varias jurisdicciones que aplican de manera conjunta ambos principios, específicamente el principio personalista para residentes fiscales y el principio de fuente para no residentes, en el caso del Ecuador ambos principios se encuentran contenidos en nuestra legislación, en el Art. 2 de la Ley de Régimen Tributario Interno.

La existencia de reglas para determinar qué jurisdicción tiene el derecho a someter a imposición la renta tiene como trasfondo el evitar la doble imposición de una misma renta y simplificar el comercio internacional. Sin embargo, por efectos del avance de la economía digital y el comercio electrónico, las transacciones comerciales se realizan en varias jurisdicciones sin la presencia física o establecimiento de la empresa en el país en el cual se realiza la transacción, lo cual ha generado varios retos para establecer quién debe ejercer su potestad tributaria en virtud de los principios tradicionales de tributación.

En virtud de esto, la OCDE ha categorizado los retos que supone la economía digital para la tributación internacional en las siguientes categorías: nexo, datos ${ }^{4}$ y caracterización ${ }^{5}$ del ingreso. Para efectos del presente artículo nos centraremos en los retos categorizados dentro de nexo.

La problemática del nexo nace por la disyuntiva sobre cómo determinar éste entre el sujeto pasivo y la jurisdicción a la que le corresponde imponer tributos sobre la renta generada, pues se cuestiona si los principios existentes permiten la determinación en función de las características específicas de los negocios digitales (OCDE, 2015).

Los modelos de negocio con influencia significativa de la tecnología tienen como característica la posibilidad de realizar transacciones comerciales en varios mercados, sin la necesidad de presencia física, ya sea a través de una sucursal, agentes, personal, entre otros. De ahí que todos los medios para la venta de bienes o prestación de un servicio se encuentran en una o varias jurisdicciones distintas a la del mercado en el que el consumidor se encuentra.

Dentro del análisis, se cuestiona si la definición de establecimiento permanente existente en la actualidad, así como los principios para atribuir a qué jurisdicción corresponde dicha renta, son aplicables a la realidad de la economía digital. Se debe considerar que la definición existente de establecimiento permanente, dentro de la tributación internacional, se encuentra recogida en los convenios para evitar la doble tributación ya sea a nivel bilateral o multilateral. Por ejemplo, el Modelo de Convenio Tributario sobre la Renta y sobre el Patrimonio (OCDE, 2019c) establece: Artículo 5 Establecimiento permanente A efectos de este Convenio, el término "establecimiento permanente" significa un lugar fijo de negocios mediante el cual una empresa realiza toda o parte de su actividad. [...]"

De acuerdo al artículo citado, el establecimiento permanente se entiende como un lugar fijo de negocios, como oficinas, sucursales, fábricas, talleres, entre otros; o la prestación de servicios por medio de agentes en dicha jurisdicción. Sin embargo, para efectos del análisis de la economía digital, la identificación del nexo entre un sujeto y la

\footnotetext{
${ }^{4} \mathrm{De}$ acuerdo a la OCDE, otro riesgo es la atribución del valor creado a partir de la generación de datos que son objeto de comercio y su impacto tributario al momento de utilizarlo para determinar el nexo.

${ }^{5}$ Así también, el riesgo de caracterización se refiere a la complejidad de la determinación de la naturaleza de la renta en función de la digitalización de la economía y las normas existentes.
} 
jurisdicción se torna complicada, en particular, por la falta de presencia física como tal o de agentes en dicho Estado y, por tanto, resulta aún más complicado determinar qué jurisdicción es aquella que tiene el derecho de atribuir a esa renta como tributable bajo su ordenamiento jurídico.

La consecuencia principal de los retos que trae la tributación de la economía digital, es la erosión de bases imponibles y el traslado de beneficios, entendido por esto la tributación a una tarifa baja o inexistente por parte de estos modelos de negocio, a través de estructuras societarias complejas y el aprovechamiento de legislaciones tributarias en jurisdicciones con sistemas tributarios poco eficientes o de baja tributación.

Una vez identificados los principales retos y riesgos de la tributación en la economía digital, es necesario determinar las posibles soluciones con base en la normativa internacional como interna.

Como se ha evidenciado, la economía digital presenta varios retos para la tributación por las características específicas de estos modelos de negocios, cuya renta debe ser objeto de tributación al igual que cualquier otra renta generada por un negocio similar por principio de neutralidad y equidad tributaria. La doctrina internacional y las legislaciones internas de ciertos países han desarrollado mecanismos a efectos de contrarrestar los retos y riesgos impositivos que supone la economía digital.

\subsection{Posibles soluciones de la OCDE}

Desde el 2013, la OCDE ha realizado varios esfuerzos para analizar y estudiar posibles soluciones para los riesgos evidenciados en la sección anterior. La OCDE ha agrupado éstas en dos pilares: el primero, se encuentra enfocado en aclarar y determinar las reglas para definir el nexo, determinar qué jurisdicción tiene la potestad tributaria, así como cuál es el valor de la renta imponible correspondiente a dicha jurisdicción; y el segundo, tiene como enfoque implementar reglas para disminuir riesgos adicionales de erosión de bases imponibles y traslado de beneficios (OCDE, 2019b).

Dentro del primer pilar, se han incluido algunas propuestas a efectos de determinar cómo se debería asignar la potestad tributaria sobre la renta generada por modelos de negocio de economía digital, que tienen como conclusión principal la atribución de mayor derecho a imponer tributos a aquella jurisdicción en la que los consumidores o usuarios se encuentren. Así, se establece un nuevo derecho para someter a imposición a la renta generada por los negocios digitales, en virtud de su participación digital, de la presencia de sus intangibles o su presencia económica e interacción con una jurisdicción a través de medios digitales (OCDE, 2019a).

En este sentido, el grupo de trabajo de la OCDE ha señalado la necesidad de establecer nuevas reglas que permitan identificar el nexo entre la jurisdicción y la empresa, separándose de la regla tradicional de la presencia física por medio de un establecimiento permanente.

Una de las propuestas consiste en que con base en el nuevo derecho para determinar quién debe imponer un tributo sobre dicha renta, la jurisdicción del mercado en el cual exista una interacción o presencia significativa será adjudicada la renta residual de la empresa para someter a imposición; atacando así los riesgos que generan aquellos modelos de negocio digitales, en particular, a aquellos relacionados con motores de búsqueda en línea, redes sociales, plataformas de intermediación o de transmisión de contenido, entre otros (OCDE, 2020).

Según la OCDE, a efectos de establecer esta forma de tributación de la economía digital, se deberán determinar indicadores que permitan identificar la presencia o interacción significativa con el mercado en una jurisdicción. 
De lo expuesto, es claro que es necesario determinar criterios objetivos que permitan a las jurisdicciones determinar cuándo se debe someter a imposición la renta de la economía digital, si bien la OCDE propone la creación de un nuevo derecho, es oportuno mencionar que el mismo podría traer más retos para las administraciones tributarias y sería mejor modificar ciertas reglas como la del establecimiento permanente, para que en función de la normativa actual se someta a imposición aquellos ingresos.

Por otro lado, el segundo pilar de la solución a plantearse por la OCDE incluye aquellas medidas que permitirán la tributación de la renta en casos en que hayan sido sometidos a imposición en jurisdicciones con tasas impositivas efectivas menores o nulas y la eliminación de deducciones como medida para aquellos pagos que erosionan la base imponible (OCDE, 2019b).

Ahora bien, es evidente que el riesgo ulterior de los retos de la tributación de la economía digital es el traslado de beneficios fuera de las jurisdicciones que se generan a unas con regímenes tributarios preferentes o con menor imposición, por lo que implementar medidas para mitigar dicho riesgo es necesario. La propuesta de la OCDE de hacerlo mediante eliminación de deducciones para los sujetos pasivos que erogan esos pagos podría ser efectiva, de la mano se podría implementar otras soluciones como la retención en la fuente al momento de realizar esos pagos, a efectos de cubrir todos los posibles riesgos; sin perjuicio de esto, cabe considerar los retos que podrían surgir para la correcta determinación de la tarifa aplicable y el posterior control de su efectivo cumplimiento.

De las soluciones expuestas por la OCDE, como parte de la búsqueda de medidas que mitiguen los retos y riesgos expuestos en la sección anterior, se puede colegir que, si bien podrían solucionar los mismos teóricamente, en la práctica se volverían insuficientes si de la mano no se implementa estrategias para la aplicabilidad de éstas por parte de las administraciones tributarias.

Sin perjuicio del trabajo de la OCDE, existen varias jurisdicciones que mediante normativa interna han intentado solventar la problemática de la tributación de la economía digital, como se expone en el siguiente apartado.

\subsection{Soluciones en la experiencia y normativa comparada}

De manera general, la normativa interna tiene como finalidad regular el sistema tributario de un Estado y generalmente las transacciones de los residentes fiscales de dicho país, entendiendo como residentes fiscales a nacionales y extranjeros que realizan actividades económicas en aquella jurisdicción. Debido a los retos de los impuestos directos, en específico, del impuesto a la renta o sus equivalentes, que generan los modelos de negocio de economía digital, existen algunas jurisdicciones que, por medio de la jurisprudencia o su normativa interna, han implementado mecanismos para mitigar los riesgos de manera unilateral.

En 2015, Reino Unido emitió una ley con la finalidad de establecer un impuesto del $25 \%$ sobre aquellos beneficios trasladados fuera del país, específicamente para no residentes de dicha jurisdicción, así la norma establece que aquellos sujetos obligados son:

86 Non-UK company avoiding a UK taxable presence

“...(c)a person ("the avoided PE"), whether or not UK resident, is carrying on activity in the United Kingdom in that period in connection with supplies of services, goods or other property made by the foreign company in the course of that trade, ...” (Finance Act, 2015).

En el mismo sentido, en el año 2017, Australia emitió una reforma tributaria con la finalidad de imponer un tributo sobre la renta del $40 \%$, con la finalidad de asegurar el pago de 
impuestos en dicha jurisdicción por entidades que tengan presencia significativa, así como con relación a una actividad económica sustancial realizada en el país, respecto de la renta trasladada fuera de éste (Diverted Protits Tax Bill, 2017).

Este tipo de normativa tiene como finalidad evitar el riesgo de erosionar la base imponible de dichas jurisdicciones por la presencia de empresas multinacionales, que regularmente no tienen un establecimiento permanente o presencia física para la ejecución de su actividad económica y por tanto genera un problema para la Administración Tributaria local al momento de ejercer su potestad tributaria.

Por otro lado, en el 2016, Nueva Zelanda incluyó en su legislación la definición de mercado electrónico, entendiendo a éste como cualquier tipo de operación por medio de la cual se provee de servicios o venta de bienes a través de medios electrónicos; situación que, sin duda, constituye un avance pues se incluye en la normativa a otros mecanismos en los cuales se pueden generar transacciones comerciales que se circunscriben en el hecho generador de un impuesto. Así, la norma dispone:

electronic marketplace-

(a) means a marketplace that is operated by electronic means through which a person (the underlying supplier) makes a supply of remote services by electronic means through another person (the operator of the market- 35 place) to a third person (the recipient); and (b) includes a website, internet portal, gateway, store, distribution platform, or other similar marketplace; and

(c) does not include a marketplace that solely processes payments. (Taxation (Residential Land Withholding Tax, GST on Online Services, and Student Loans) Bill, 2016).

Por otro lado, a nivel regional, la legislación peruana ha incluido como ingreso de fuente peruana a la renta obtenida por servicios digitales, siempre que el uso o consumo del mismo suceda en el país. La Ley del Impuesto a la Renta, en el artículo 9 dispone:

Artículo $9^{\circ}$.- En general y cualquiera sea la nacionalidad o domicilio de las partes que intervengan en las operaciones y el lugar de celebración o cumplimiento de los contratos, se considera rentas de fuente peruana:

i) Las obtenidas por servicios digitales prestados a través del Internet o de cualquier adaptación o aplicación de los protocolos, plataformas o de la tecnología utilizada por Internet o cualquier otra red a través de la que se presten servicios equivalentes, cuando el servicio se utilice económicamente, use o consuma en el país (2004).

En este sentido, es claro que varias jurisdicciones alrededor del mundo han dado importantes pasos a efectos de establecer soluciones impositivas respecto de los riesgos que genera la economía digital, tanto respecto a la dificultad de su tributación efectiva, así como aquellos respecto de la erosión de bases imponibles y el traslado de beneficios.

Si bien de los ejemplos de normativa comparada expuestos no se puede concluir que solucionan de manera completa todos los retos y riesgos de la tributación de la economía digital, considero que existen ciertos elementos que podrían incorporarse dentro de la legislación ecuatoriana para iniciar a contrarrestar los riesgos de estos modelos de negocios, conforme se reseñará en los apartados siguientes. 
Además de las soluciones internacionales de la OCDE y aquellas de cada jurisdicción existen otras planteadas por la academia como imponer una retención en la fuente a nivel mundial respecto de este tipo de negocios, lo cual evidentemente requeriría la modificación del Modelo Convenio de la OCDE así como de mecanismos para la exigibilidad del mismo (Brauner y Báez, 2015). La presente solución si bien es teóricamente aplicable y sostenible en el tiempo, la implementación de la misma - por su exigibilidad - será compleja pues implicaría la suscripción o adhesión mundial de las jurisdicciones a un instrumento multilateral que seguramente tomará tiempo.

En virtud de lo expuesto, la normativa comparada y la academia han brindado ciertas alternativas para actuar frente a los retos y riesgos de la economía digital, por lo que se deberá analizar cuál es la situación en nuestro país.

\section{4. ¿Cuál es la situación de la tributación de la economía digital en Ecuador?}

La Constitución de la República del Ecuador ha establecido que el régimen tributario se regirá por varios principios, ${ }^{6}$ entre ellos, el de equidad. A criterio de la OCDE, este principio es fundamental al momento del desarrollo de la política y normativa tributaria como a efectos de contrarrestar aquellos riesgos que genera la economía digital en los regímenes fiscales.

Así, la OCDE ha establecido que la equidad dentro de su análisis sobre economía digital tiene una trascendental incidencia pues se debe entender que aquellos contribuyentes que se encuentren en circunstancias similares deberán tener carga tributaria similar (2015, p. 21). En este sentido, aquellos agentes económicos que realizan actividades a través de medios tecnológicos deberían tributar en igual forma que aquellos que lo realizan a través de otro tipo de medios con presencia física.

La normativa tributaria ecuatoriana no ha presentado mayor desarrollo frente a la tributación de la economía digital, en particular, en cuanto a impuestos directos como el impuesto a la renta. Sin perjuicio de esto, a finales de 2019 por medio de la Ley Orgánica de Simplificación y Progresividad Tributaria se reformó la Ley de Régimen Tributario interno incluyendo como parte del hecho generador del Impuesto al Valor Agregado a los servicios digitales.

Ahora bien, cabe analizar si las soluciones de la OCDE serían aplicables bajo el marco normativo ecuatoriano; si la legislación ecuatoriana, respecto de impuesto a la renta de sociedades, tiene algún mecanismo normativo a efectos de absolver aquellos retos de la tributación de los modelos de negocio digitales; y, por último, qué otras opciones tendría el Ecuador para ejercer un mayor control.

Es preciso recordar que el riesgo principal que trae la economía digital es la erosión de bases imponibles y el traslado de beneficios pues dichos modelos de negocio, por las características antes expuestas, suelen generar una tributación nula o a tasas mínimas; situación que deviene en retos adicionales respecto a cómo determinar la jurisdicción que tiene el derecho para someter a imposición la renta y bajo qué mecanismos por la falta de presencia física de los agentes económicos.

La OCDE planifica tener una solución consensuada a finales del 2020, la cual establecerá nuevas reglas que permitirán determinar la jurisdicción que tiene el derecho

\footnotetext{
${ }^{6}$ Los principios que rigen al sistema tributario son generalidad, progresividad, eficiencia, simplicidad administrativa, irretroactividad, equidad, transparencia y suficiencia recaudatoria (artículo 300).

${ }^{7}$ El Reglamento a la Ley de Simplificación y Progresividad Tributaria, publicado el 4 de agosto de 2020, definió a los servicios digitales entendidos como aquellos a los prestados y/o contratados a través de internet, o de cualquier adaptación o aplicación de los protocolos, las plataformas o la tecnología utilizada por internet, u otra red a través de la que se presten servicios similares que, por su naturaleza, estén básicamente automatizados y requieran una intervención humana mínima, independientemente del dispositivo utilizado para su descarga, visualización o utilización.
} 
para ejercer su potestad tributaria, así como nuevas reglas para establecer el nexo entre el agente económico y la jurisdicción. Éstas modificarán las reglas tradicionales, en lo principal, eliminando la presencia física en la jurisdicción como elemento necesario. Sin perjuicio de esto, se debe considerar que, si bien la normativa de la OCDE tiene efectos vinculantes a nivel internacional, Ecuador no es miembro de dicha organización y por tanto las directrices emitidas de manera general no son vinculantes para nuestro país, sino solo referenciales.

A pesar de esto, es claro que las directrices que la OCDE emita respecto a la tributación de la economía digital serán aplicables para los países miembros ${ }^{8}$ y conllevarán modificaciones al Modelo de Convenio Tributario sobre la Renta y sobre el Patrimonio, lo cual generará un efecto dominó pues los países miembros se verán de igual forma en la necesidad de modificar o incluir las provisiones correspondientes en los convenios para evitar la doble tributación suscritos bilateralmente.

\subsection{1. ¿Qué mecanismos tiene la legislación ecuatoriana para afrontar los retos de la economía digital?}

Conforme se reseńó previamente, la normativa tributaria ecuatoriana no tiene ninguna previsión específica respecto de la tributación de la economía digital, en términos del Impuesto a la Renta, tal es así que si bien para efectos impositivos el Ecuador considera como sujetos pasivos de impuesto a la renta a todas las sociedades ${ }^{9}$ ecuatorianas o extranjeras, dicha imposición resulta impráctica.

Ahora bien, respecto de las sociedades extranjeras y por tanto no residentes en el Ecuador, éstas pueden ser sujetos pasivos del impuesto a la renta de sociedades cuando tengan un establecimiento permanente en Ecuador, mismo que por definición reglamentaria es el lugar fijo para la realización de actividades. ${ }^{10}$ Es decir, que bajo la normativa ecuatoriana se mantiene el mismo problema frente a la tributación digital, pues la definición de establecimiento permanente se circunscribe a la presencia física en el país. Así, los establecimientos permanentes tributan bajo el sistema tradicional de impuesto a la renta en el Ecuador, como una sociedad y bajo la tarifa prescrita en el artículo 36 de la Ley de Régimen Tributario Interno.

Sin perjuicio de esta forma de tributación, la normativa tributaria ecuatoriana también establece de manera categórica que todos los no residentes tributarán ${ }^{11}$ sobre los ingresos generados en el Ecuador a la misma tarifa prevista para sociedades $(25 \%)$ y a la tarifa máxima de impuesto a la renta para personas naturales (35\%), si el no residente tiene residencia fiscal en un paraíso fiscal, jurisdicción de menor imposición o régimen fiscal preferente, conforme lo prescrito en el artículo 39 de la Ley de Régimen Tributario Interno. Dicha imposición se realiza a través de retención en la fuente por parte del sujeto que realiza el pago.

\footnotetext{
${ }^{8}$ Actualmente los siguientes 37 países son miembros de la OCDE: Australia, Austria Bélgica, Canadá, Chile, Colombia, República Checa, Dinamarca, Estonia, Finlandia, Francia, Alemania, Grecia, Hungría, Islandia, Irlanda, Israel, Italia, Japón, Corea, Letonia, Lituania, Luxemburgo, México, Países Bajos, Nueva Zelanda, Noruega, Polonia, Portugal, Eslovenia, Espańa, Suecia, Suiza, Turquía, Reino Unido, Estados Unidos.

${ }^{9}$ Cabe considerar que, de conformidad con la Ley de Régimen Tributario Interno, se considera sociedad a toda persona jurídica, sociedad de hecho, fideicomiso mercantil y en general cualquier entidad que, aunque carente de personería jurídica, constituya una unidad económica o un patrimonio independiente de los de sus miembros.

${ }^{10}$ El Reglamento para la aplicación a la Ley de Régimen Tributario Interno, tiene una definición de establecimiento permanente similar a la del Modelo de Convenio Tributario sobre la Renta y sobre el Patrimonio de la OCDE.

${ }^{11}$ El artículo 39 de la Ley de Régimen Tributario Interno establece dicha obligación y la tarifa diferenciada a la que tributarán según su residencia fiscal los no residentes.
} 
En virtud de lo expuesto, se podría concluir entonces que la legislación ecuatoriana no resuelve como tal todos los retos que trae la tributación de la economía digital pues si bien la normativa interna parecería ser clara respecto de la imposición sobre los ingresos percibidos por no residentes, existen muchas otras aristas no cubiertas por la norma, tales como la imposición a no residentes sin establecimiento permanente o presencia física en el país, aquellos casos en el que no existe un intermediario para el pago, como las empresas emisoras de tarjetas de crédito o débito o definitivamente el sujeto que paga no se constituye como un agente de retención.

En primera instancia, la norma es efectiva cuando existe un agente de retención y cuando hay una transacción entre dos sujetos un residente y un no residentes de manera directa. Por ejemplo, si una compañía paga por un servicio a una compañía del exterior, independientemente de su domicilio, deberá realizar la retención en la fuente respectiva, generando la tributación del ingreso del no residente, siempre que no exista un régimen específico aplicable o un convenio para evitar la doble imposición entre ambas jurisdicciones. Sin embargo, la normativa local ecuatoriana prescribe tratamientos específicos para ciertas transacciones con sujetos del exterior; y sin perjuicio de esto, el reto principal de la imposición resulta complejo cuando el sujeto que paga no es un agente de retención o en aquellos casos en el que intermediario para el pago no residen en el Ecuador.

Incluso cuando el pago es realizado por medio de una tarjeta de crédito y por tanto trasladándose la obligación como agente de retención a la institución financiera, la normativa no es eficaz para los negocios de economía digital, pues al no existir presencia física de la empresa no existiría establecimiento afiliado en los términos de la normativa vigente para que medie la retención en la fuente, y en los casos en los que se genera la retención en la fuente, en la práctica no resulta aplicable por la forma en cómo se realiza operatividad en los pagos por los emisores de tarjetas de crédito o débito.

Finalmente, uno de los mayores retos para la Administración Tributaria tanto a nivel local, como en otras jurisdicciones, es el control de la tributación de los no residentes en el país, pues dicho control, por los problemas operativos y aristas que hemos visto previamente resultaría muy complejo con la estructura y normativa vigente.

En este sentido, la normativa ecuatoriana respecto de la tributación de no residentes fiscales no contrarresta la imposición de economía digital, pues el ámbito de aplicación de la Ley de Régimen Tributario Interno, no incluye normas respecto de estos agentes económicos como sujetos pasivos del tributo, en términos que resulte realmente aplicable por la forma de cómo se realizan este tipo de negocios, si bien existe una norma general de imposición a no residentes, conforme se analizó previamente existen varios retos para efectos de su real aplicabilidad y control de la Administración Tributaria

\subsubsection{1 ¿Qué alternativas existen para mitigar los riegos de la tributación de la economía digital por medio de normativa interna?}

Con base en la problemática descrita, resulta evidente que para que Ecuador pueda tener mecanismos efectivos para contrarrestar los retos tributarios de la economía digital, evitando la erosión de bases imponibles y traslado de beneficios, se podrían analizar varias alternativas enfocadas desde varias aristas, por un lado constituir a los prestados de servicios o proveedores de bienes de la economía digital como establecimientos permanentes; o, por otro lado, establecer un mecanismo de retención en la fuente más efectivo, entre otras.

Respecto de la primera alternativa, la legislación ecuatoriana debería comenzar con la ampliación de la definición de establecimiento permanente a efectos de incluir aquellas 
definiciones que incorporan la presencia digital de los sujetos pasivos y de tal forma incluir también a estos sujetos dentro de su normativa de tributación de no residentes. Así, se propone una reforma al Reglamento para la Aplicación de la Ley de Régimen Tributario Interno, creando un literal c) en el numeral 1 del artículo 9:

Art. 9.- Establecimientos permanentes de empresas extranjeras.-

1. Para efectos tributarios, establecimiento permanente es el lugar en el que una empresa efectúa todas o parte de sus actividades.

Bajo tales circunstancias, se considera que una empresa extranjera tiene establecimiento permanente en el Ecuador, cuando:

c) Preste servicios o venda bienes cuyo uso o consumo se realice en el Ecuador, a través de medios digitales, entendiéndose medios digitales aquellos que utilicen como canales para realizar la transacción cualquier plataforma, red o aplicación a través de internet.

Adicionalmente, es recomendable que el Ecuador realice una reforma respecto de qué se considera ingresos de fuente ecuatoriana similar a la realizada por la legislación peruana que incluye como renta la proveniente de servicios digitales que se usen o consuman en dicha jurisdicción; dicha reforma en conjunto con la modificación de la definición de establecimiento permanente y la generación de la tributación por medio de retención en la fuente por el sujeto pasivo ecuatoriano que realiza el pago, podría mitigar los riesgos tributarios de la economía digital.

Ahora bien, considerando que la recaudación, así como el control de estos sujetos pasivos será en definitiva un problema, pues implicaría una aplicación extraterritorial de la ley tributaria, existen académicos que han establecido que dicho problema se puede solucionar por medio de acuerdos bilaterales o multilaterales (Hongler y Pasquale, 2015). Además cabe considerar que existen mecanismos en los convenios suscritos por el Ecuador para la asistencia en el cobro de impuestos. ${ }^{12}$ Así también, una vez determinadas las directrices de la OCDE respecto a la materia, podría implementarlas legalmente tal y como lo ha hecho en otras materias, precios de transferencia, por ejemplo.

Por otro lado, desde la publicación de la Ley Orgánica de Simplificación y Progresividad Tributaria, el Ecuador estableció varias normas reglamentarias a efectos de materializar la imposición de los servicios digitales respecto del Impuesto al Valor Agregado, tanto en la prestación local como en la importación de éstos. Así, la normativa vigente ha dispuesto la publicación de un catastro de prestadores de servicios digitales, mismo que incluye a aquellos residentes como no residentes en el país a efectos de la operatividad y el control la retención por dicho impuesto.

A efectos de implementar la tributación del Impuesto al Valor Agregado para servicios digitales, la Administración Tributaria ha establecido la retención en la fuente a cargo de los sujetos pasivos que realicen el pago o a través de su intermediario.

En este sentido, se debe considerar que la normativa emitida por concepto del Impuesto al Valor Agregado, es un avance para la tributación de la economía digital y podría resultar beneficioso para el siguiente paso que sería la tributación en impuesto a la renta.

Otra de las alternativas dentro del impuesto a la renta, sería generar una retención en la fuente a los bienes y servicios objeto de transacciones de economía digital, misma que debería distar de aquella genérica de pagos al exterior, aprovechando en primera instancia el catastro y

\footnotetext{
${ }^{12}$ El Ecuador como signatario de la Convención Multilateral sobre Asistencia Administrativa Mutua en Materia Fiscal puede acceder a la asistencia en el cobro de créditos fiscales a otros países miembros.
} 
la información que la Administración Tributaria ya ha ido recabando por efectos del Impuesto al Valor Agregado. La importancia de la distinción con la retención general de ingresos de no residentes, es para efectos de facilitar la aplicación de la misma y que en efecto se genere una imposición de los beneficios obtenidos por no residentes, respecto de aquellos bienes y servicios que son comercializados en el Ecuador.

\section{Conclusiones}

Conforme lo expuesto en el presente artículo, es claro que existen varios riesgos en la imposición de la economía digital para los Estados, pues la principal contingencia es si existe o no una tributación efectiva respecto de dichos ingresos que perciben en distintos lugares en razón del domicilio de dichos sujetos y la tasa impositiva efectiva en aquellas jurisdicciones.

Es claro que la renta que perciben los sujetos que intervienen en la economía digital, deben tributar en la misma forma y cuantía que lo haría cualquier sujeto pasivo de impuesto a la renta que tendría la misma actividad económica, pero sin la presencia de mecanismos digitales como enfoque principal de su negocio.

Así, del análisis realizado es evidente que la legislación ecuatoriana carece de mecanismos efectivos a efectos de absolver los retos sobre la tributación de la economía digital, pues sus previsiones generales y, en específico, aquellas relativas al establecimiento permanente, están centradas en la presencia física como nexo para la imposición en nuestro país.

En este sentido, si bien una de las posibles soluciones a la tributación de la economía digital podría ser la ampliación de la definición del establecimiento permanente, se debe enfocar el criterio de sujeción de dichos sujetos a aquellos bienes vendidos o servicios prestados en el Ecuador, a través de mecanismos digitales. Sin perjuicio de que dichos sujetos ya no se considerarían como no residentes fiscales en el país, tributarían como residentes fiscales ecuatorianos únicamente sobre aquellos beneficios que perciban por sus transacciones en esta jurisdicción.

Por otro lado, conforme lo expuesto el Ecuador no evidencia ningún avance de incluir normativa respecto de la tributación de economía digital en impuesto a la renta, pero con las normas recientemente emitidas respecto de la tributación de servicios digitales con relación al Impuesto al Valor Agregado, se podría aprovechar dichos elementos para regular el mismo aspecto en un esquema de tributación directa, en el impuesto a la renta.

Finalmente, es claro que los negocios con influencia de tecnología son crecientes en nuestro país y más aún como efecto colateral de la pandemia en razón del Covid-19; por lo que, en el futuro cercano se deberá comenzar a incluir normativa como la propuesta anteriormente, que regule estas situaciones de hecho, que de la mano con la normativa existente respecto a tributación de no residentes podría mitigar los riesgos para nuestro país a fin de generar una tributación efectiva y equitativa de este tipo de modelos de negocio.

\section{Referencias bibliográficas}

Altenburger, P y Czajkowski, J. (2020). How to Tax the Digital Economy? Novità fiscali, 2020 (4), 208-214.

Altenburger, P y Czajkowski, J. (2020). How to Tax the Digital Economy? Novità fiscali, 2020 (4), 208-214. 
Brauner, Y. y Baez, A. (2015). Withholding Taxes in the Service of BEPS Action 1: Address the Tax Challenges of the Digital Economy. WU International Taxation Research Paper Series No. 2015 - 14 SSRN-id2591830

Borderas, E. Moles, P. (2008). Derecho Tributario Internacional. Barcelona: Editorial el Fisco G.L.E.T. S.L. ISBN: 978-84-933400-4-9

Hongler, P. y Pasquale, P. (2015). Blueprints for a New PE Nexus to Tax Business Income in the Era of the Digital Economy. Working paper 20 January 2015. IBFD - SSRN: https:// ssrn.com/abstract $=2591829$

Li, J. (2018). Protecting the Tax Base in a Digital Economy. Osgoode Legal Studies Research Paper, 13 (78), 17.

Johnston, D. (2001) E-Business and Digital Economy: The Policy Challenge. Baltic IT\&T Review. Recuperado el 22 de mayo de 2020, de: http://www.OCDE.org/internet/ ieconomy/2350363.pdf

Martínez, C. (2016). Impuestos Directos e Indirectos en la Argentina: Su relación con el desempeño económico. Estudios Económicos, 33(66), 47-64.

Massachusetts Institute of Technology. (2014). How to Win with a Multisided Platform Business Model. Recuperado 24 de mayo de 2020, de https://sloanreview.mit.edu/article/howto-win-with-a-multisided-platform-business-model/

OCDE (2015), Addressing the Tax Challenges of the Digital Economy, Action 1 - 2015 Final Report. Paris: OCDE/G20 Base Erosion and Profit Shifting Project, http://dx.doi. org/10.1787/9789264241046-en

- (2019a) Public Consultation Document, Addressing the Tax Challenges of the Digitalisation of the Economy, 13 February - 6 March 2019. París: OCDE/G20 Inclusive Framework on BEPS, OCDE, https://www.OCDE.org/tax/beps/public-consultation-documentaddressing-the-tax-challenges-of-the-digitalisation-of-the-economy.pdf

- (2019b), Programme of Work to Develop a Consensus Solution to the Tax Challenges Arising from the Digitalisation of the Economy. París: OCDE/G20 Inclusive Framework on BEPS, OCDE, www.OCDE.org/tax/beps/programme-of-work-to-develop-a-consensus-solution-to-thetax-challenges-arising-from-the-digitalisation-of-the-economy.html

- (2019c), Modelo de Convenio Tributario sobre la Renta y sobre el Patrimonio: Versión Abreviada 2017, OCDE Publishing, Paris/Instituto de Estudios Fiscales, Madrid, https://doi. org/10.1787/765324dd-es.

- (2020), Statement by the OCDE/G20 Inclusive Framework on BEPS on the Two-Pillar Approach to Address the Tax Challenges Arising from the Digitalisation of the Economy - January 2020, París: OCDE/G20 Inclusive Framework on BEPS, OCDE, http:// www.oecd.org/tax/beps/statement-by-the-oecd-g20-inclusive-framework-on-bepsjanuary-2020.pdf

Olbert, M. Spengel, C. (2017). International Taxation in the Digital Economy: Challenge Accepted? World Tax Journal. 9 (1), 3-46.

Ormaza, D. Vázquez, A. (2019). La potestad tributaria en el Derecho Internacional Tributario Revista Killkana Sociales. 3(3), 39-44. https://doi.org/10.26871/killkana_social. v3i3.206

Soto, M. (2017). Derecho Tributario Internacional. México: Instituto Mexicano de Contadores Públicos A.C.

Tax Policy Center (2020). Key Elements of the U.S. Tax System. Recuperado el 13 de mayo de 2020, de: https://www.taxpolicycenter.org/briefing-book/what-territorial-tax-anddoes-united-states-have-one-now 
Troya, J. (2014). Manual de Derecho Tributario. Quito: Corporación de Estudios y Publicaciones. Universidad Espíritu Santo (2019). Comportamiento de las Transacciones No Presenciales en Ecuador. Recuperado 21 de mayo de 2020, de http://www.cece.ec/

\section{Legislación}

Asamblea Constituyente (2008). Constitución de la República del Ecuador. Registro Oficial No. 449 de 20 de octubre de 2008.

Asamblea Nacional de la República del Ecuador (2019). Código de Comercio. Registro Oficial Suplemento No. 487 de 29 de mayo de 2019.

Congreso de la República del Perú (2004). Ley del Impuesto a la Renta. Decreto Supremo N. ${ }^{\circ}$ 179-2004-EF de 8 de diciembre de 2004.

Congreso Nacional (2002). Ley de Comercio Electrónico, Firmas Electrónicas y Mensajes de Datos. Registro Oficial Suplemento No. 557 de 17 de abril de 2002.

Congreso Nacional (2004). Ley de Régimen Tributario Interno. Registro Oficial Suplemento No. 463 del 17 de noviembre de 2004.

New Zeland Parliament (2016). Taxation (Residential Land Withholding Tax, GST on Online Services, and Student Loans) Bill, Nueva Zelanda, 13 de mayo de 2016.

Parliament of the United Kingdom (2015). Finance Act 2015, Reino Unido, 26 de marzo de 2015.

Parliament of Australia (2017). Diverted Profits Tax Bill, Australia, 04 de abril de 2017.

Presidente Constitucional de la República (2020). Reglamento para la Aplicación de la Ley Orgánica de Simplificación y Progresividad Tributaria. Registro Oficial Suplemento No. 260 del 4 de agosto de 2020.

Servicio de Rentas Internas (2020). Resolución No. NAC-DGERCGC20-00000053. Establecer las normas para la retención del Impuesto al Valor Agregado (IVA) generado en la importación de servicios digitales cuando los prestadores del servicio importado no actúen como agentes de percepción. Registro Oficial Edición Especial No. 920 del 25 de agosto de 2020. 